\title{
IMPLEMENTATION OF THE AXIOMS OF COSSERAT-ZHILIN SYSTEMS ON EXAMPLES OF THEIR MAIN TYPES
}

\author{
Al. Cheremensky* \\ Institute of Mechanics, Bulgarian Academy of Sciences, Sofia 1113, Bulgaria
}

[Received 25 November 2016. Accepted 08 October 2018]

doi: 10.7546/JTAM.49.19.01.01

\begin{abstract}
The differential equations of motion are derived for different types of Cosserat-Zhilin systems in the case where Stocks theorem is applicable. There are defined multiplicative groups which represent the measure of stress as a (well-defined) linear isotropic map of strain tensor or tensor of strain velocities for classical and polar continua in 2- and 3-dimensional cases.
\end{abstract}

KEY WORDS: Classical mechanics, continuum mechanics, mechanical measures, foundations of mechanics, constitutive equations, screw theory.

\section{INTRODUCTION}

The goal of Newton was to give an answer to the question whether there is a simple rule for calculating the total movement of the heavenly bodies of our planetary system at a given state of motion of all the bodies in a given time? From observations of Tycho Brahe, Kepler deduced empirical laws of planetary motion but they were demanded an explanation. Today, everyone knows what a great, truly bee, hard work has been required to establish these laws, on the basis of empirically determined orbits. But few who imagines the genius of the method by which Kepler has defined the true orbit, based on the apparent, i.e., of the observed motions of the Earth. These laws provide a complete description of the motion of the planets around the Sun: elliptical orbits, equality sectorial velocity ratio between the semi-major axes and periods of treatment. But these laws do not satisfy the requirement of a causal explanation. They were the three logically independent of each other rules deprived of any internal connection. The third law cannot be quantified unequivocally transferred to another, other than the Sun, the central body (there is, for example, no connection between the orbital period of the planet around the Sun and the orbital period of the satellite around its planet). But the important thing is that the laws of motion are generally not possible to

\footnotetext{
${ }^{*}$ Corresponding author e-mail: cheremensky@yahoo.com
} 
derive from the state of motion at some point in time a different state in time immediately following the first. In modern terminology, we would say that they are integral laws and not differential.

Differential law is the sole form of causal explanation, which can fully meet modern physics. A clear understanding of the differential law is one of the greatest intellectual achievements of Newton [1].

In this connection, we are about to show the implementation of the axioms on examples of main types of Cosserat-Zhilin systems and derive their equations of motion in the case where Stocks theorem is applicable ( $\left.\operatorname{see}^{1}[3]\right)$. These equations are invariants of the generalized Galilean group (see Appendix C and [4]).

\section{BODY-POINT}

Let the image of a world-line $\boldsymbol{\Lambda} \subset \tilde{\boldsymbol{\Lambda}}$ be the curve $\left\{x(t) \in \Lambda_{t}, t \in \mathbf{T}\right\}$ in $\mathbf{R}^{3} \times \mathbf{T}$. Assume that the points $x$ of $x(t)$ are pure. Then the corresponding mechanical system is called body-point ${ }^{2}$. For body-points, in the case (5, [2]), from relations (3, [2]) and $(6,[2])$ follows that:

(1) $\frac{d}{d t} \rho_{x} l^{\tilde{p}_{x}, \tilde{q}_{x}}=l^{\alpha_{x}, \beta_{x}}, \quad\left(\begin{array}{c}\tilde{p}_{x} \\ \tilde{q}_{x}\end{array}\right)=\left[\begin{array}{cc}I & A \\ A^{T} & B\end{array}\right]\left(\begin{array}{c}v_{x} \\ \mu_{x}\end{array}\right), \quad x \in x(t) \subset \Lambda_{t}, t \in \mathbf{T}$

or (see also Appendix B)

$$
\left(\rho_{x} \frac{d}{d t}+\nu_{x}\right)\left[\begin{array}{cc}
I & A \\
A^{T} & B
\end{array}\right]\left(\begin{array}{c}
v_{x} \\
\mu_{x}
\end{array}\right)=\left(\begin{array}{c}
\alpha_{x} \\
\beta_{x}
\end{array}\right), \quad x \in x(t) \subset \Lambda_{t}, t \in \mathbf{T} .
$$

Remark 1. In physics, if a body has an infinitely small size and a finite mass, it is called a material point or mass-point. We could put an electron on the role of material point because its size is extremely small, and it has some mass. However, motion of electrons can be not only translational, but also rotational. The latter does not meet the concept of material point (its rotation is not defined). Thus, a priori, we cannot consider a body with an infinitely small size and a finite mass as a material point. That is why we hope that the motion of an electron may be described as that of a body-point with finite mass and charge, i.e. when

$$
\theta_{x} \stackrel{\text { def }}{=}\left[\begin{array}{cc}
I \rho_{x} & A q_{x} \\
A^{T} q_{x} & B
\end{array}\right],
$$

where $q_{x}$ is so-called 'charge' [6]. The free motion of such a mechanical system may not be uniform and rectilinear.

\footnotetext{
${ }^{1}$ Here, we continue to use the numbering of bibliography, sections and relations as given in [2].

${ }^{2}$ This concept is not the same as in [5] (see also [6]).
} 


\section{Polar Medium}

We shall assume that all points of $\Lambda_{t} \subset \tilde{\Lambda}_{t}$ are continuous and $\Lambda_{t}$ has the surface $\partial \Lambda_{t}$ which is Lyapunov's simple closed one (see also [6]).

Introduce so-called measure $\mathcal{F}$ of mass action, having the values

$$
\mathcal{F}\left(\Lambda_{t}\right)=\int \chi_{\Lambda_{t}} \rho_{x} l^{\gamma_{x}, \delta_{x}, w r} \mu_{3}(d x), \quad \Lambda_{t} \subset \tilde{\Lambda}_{t}, t \in \mathbf{T} .
$$

Let constraints being in a small vicinity of $x \in \Lambda_{t}$ cause the measure of stress [7] having the values

$$
\mathcal{R}_{\text {int }}\left(\Lambda_{t}\right)=\int \chi_{\partial \Lambda_{t}} l^{P x, Q_{x}, w r} n_{x} \mu_{2}(d x), \quad \Lambda_{t} \subset \tilde{\Lambda}_{t}, t \in \mathbf{T} .
$$

Hereinafter $\mu_{2}$ is the restriction of $\mu_{3}$ on the surface $\partial \Lambda_{t}, n_{x}$ is the normal to this surface; $P_{x}$ and $Q_{x}$ are 2 nd order tensors.

Definition 1. The mechanical system $\alpha=\left\{\Lambda_{t} \subset \tilde{\Lambda}_{t}, \mu_{3}, \nu_{x}, \theta_{x}, \mathcal{F}, \mathcal{R}_{\text {ext }}, \mathcal{R}_{\text {int }}, \forall t \in\right.$ T\} is called polar medium [8].

For the sake of brevity assume that $\mathcal{R}_{\text {ext }} \equiv 0$.

Lemma 1. Let the tensor-function $P_{x}$ be continuously differentiable in the fit region $\Lambda_{t}$. Then [9]

$$
\int \chi_{\Lambda_{t}} \operatorname{div}\left(R_{y, x} P_{x}\right) \mu_{3}(d x)=\int \chi_{\Lambda_{t}}\left(R_{y, x} \operatorname{div} P_{x}+\tau_{x}\right) \mu_{3}(d x), \quad \Lambda_{t} \subset \tilde{\Lambda}_{t}, t \in \mathbf{T},
$$

where the skew 2 nd order tensor $R_{y, x}$ is generated by the translation vector $r_{y, x}$ : $R_{y, x}=r_{y, x}^{\times} ; \tau_{x}$ is the dual vector to $P_{x}^{T}-P_{x}$ (see Appendix A [2]).

In the case where Stocks theorem is applicable, due to lemmas 1-3 [2], from relation (6, [2]) follows (see Appendix B [2])

$$
\left(\rho_{x} \frac{d}{d t}+\nu_{x}\right)\left[\begin{array}{cc}
I & A \\
A^{T} & B
\end{array}\right]\left(\begin{array}{c}
v_{x} \\
\mu_{x}
\end{array}\right)=\rho_{x}\left(\begin{array}{c}
\gamma_{x} \\
\delta_{x}
\end{array}\right)+\operatorname{div}\left(\begin{array}{c}
P_{x} \\
Q_{x}
\end{array}\right)+\left(\begin{array}{c}
o \\
\tau_{x}
\end{array}\right),
$$

where $o$ is the null vector.

Take a point $y(t)$ in a small vicinity of $x(t) \in \Lambda_{t}$ at an instant $t \in \mathbf{T}$ and define their radius-vectors $r_{x}$ and $r_{y}$ and the vector $h(t)=r_{y}-r_{x}$. Then there is the following relation:

$$
v_{y}(t) \cong v_{x}(t)+d v_{x} / d r_{x} h(t) .
$$

Define the tensor $S_{x}(t)$ as the solution of the following equation:

$$
S_{x}^{*}(t)=d v_{x} / d r_{x}
$$

where its initial data are defined by so-called deformed state of the medium. 
Definition 2. $S_{x}$ and $S_{x}^{\cdot}$ are called strain tensor and tensor of strain velocities at the point $x \in \Lambda_{t}$ at the instant $t$, respectively [4].

Denote the tensor $S_{x}$ or $S_{x}^{*}$ as $Z_{x}$.

Definition 3. The polar medium is called that of Hooke class if the tensors $P_{x}$ and $Q_{x}$ are linear isotropic maps of $Z_{x}$, i.e., invariant w.r.t. Galilean group of transformations (see also Appendix A).

Remark 2. If $Z_{x}=S_{x}$ the medium is called elastic material, if $Z_{x}=S_{x}^{*}$ the medium is called viscous fluid [10].

Definition 4. We shall call an isotropic matrix function of entries of $Z$ well-defined if it is invertible (see also [4]).

The set of invertible linear isotropic matrix functions forms a multiplicative group (see Appendix B).

Regarding the implementation of polar media - see, e.g., $[3,9,11]$ and Appendix C.

\section{MASS-POINT}

In what follows, we shall assume that $A$ and $B$ are zero in relation (5, [2]), i.e., we shall use homogeneous sliders and the measure $\mathcal{P}$ having the values

$$
\mathcal{P}\left(\Lambda_{t}\right)=\int \chi_{\Lambda_{t}} \rho_{x} l^{v_{x}} \mu_{3}(d x), \quad \Lambda_{t} \subset \tilde{\Lambda}_{t}, t \in \mathbf{T} .
$$

Assume that the increment velocity of $\mathcal{P}$ is the measure $\Delta \mathcal{P}$ having the values

$$
\Delta \mathcal{P}\left(\Lambda_{t}\right)=\int \chi_{\Lambda_{t}} l^{\xi_{x}} \mu_{3}(d x), \quad \Lambda_{t} \subset \tilde{\Lambda}_{t}, t \in \mathbf{T},
$$

where the slider $l^{\xi_{x}}$ is its density.

Consider a world-line $\boldsymbol{\Lambda} \subset \tilde{\boldsymbol{\Lambda}}$ whose image in $\mathbf{R}^{3} \times \mathbf{T}$ generates the curve $\{x(t) \in$ $\left.\Lambda_{t}, t \in \mathbf{T}\right\}$. Assume that the points $x(t)$ are pure.

Let $f_{x}$ be the impressed force acting at the point $x=x_{k} \in \Lambda_{t}$ with the mass $\mathcal{M}=\rho_{x} \mu_{p p}$. Then the mechanical system $\alpha=\left\{x(t) \in \Lambda_{t}, \mu_{p p}, f_{x}, \nu_{x}, \rho_{x}, \xi_{x}\right.$, $\forall t \in \mathbf{T}\}$ is called mass-point.

From relation (6, [2]) follows that:

$$
\left(\rho_{x} \frac{d}{d t}+\nu_{x}\right) v_{x}=f_{x}+\xi_{x} .
$$

If $\nu_{x} \equiv 0$ and $\xi_{x} \equiv 0$, then equation (3) is known as Newton's Second Law.

If $\nu_{x} \neq 0$ and $\xi_{x}=\nu_{x} u_{x}$, where $u_{x}$ is the velocity of mass gain or loss, then equation (3) is known as that of Meshchersky [12].

Remark 3. A classical example of mass-points with constraints is the mechanical system known as pendulum. 


\section{RIGID BODY}

For the sake of brevity assume that $\Delta \mathcal{P}$ and $\mathcal{R}_{\text {ext }} \equiv 0$.

The mechanical system $\alpha=\left\{\Lambda_{t} \subset \tilde{\Lambda}_{t}, \mu_{3}, \rho_{x}, \mathcal{F}, \mathcal{R}_{\text {int }}, \forall t \in \mathbf{T}\right\}$ is called rigid $b o d y^{3}$ if

- the sets $\Lambda_{t}$ are bounded and closed;

- the constraints applied on its points keep distances between them not changing with time;

— the internal constraints are ideal [13].

Let us derive its motion equation with using so-called quasi-velocities (NewtonEuler equation), some parametrizations of rotation matrices and generalized coordinates and velocities (Lagrange equation of II kind).

Newton-Euler equation. At any time instant $t^{*}$ consider the set $\Lambda_{t^{*}}$. Let a Cartesian frame $\mathcal{E}_{p}$ be attached to the set under consideration. It is plain that the frame takes the same position in all sets $\Lambda_{t}$. In the frame these sets are immobile, coincide one with another and form the set noted as $\Lambda_{p}$ in the frame $\mathcal{E}_{p}$. We shall say that the frame $\mathcal{E}_{p}$ is attached to the rigid body $\alpha_{p}$. Let $v_{0, p}$ and $\omega_{0, p}$ be the translation velocity and the angular velocity of $\mathcal{E}_{p}$ w.r.t. $\mathcal{E}_{0}$.

It is plain that the vectors $\omega_{0, p}$ and $v_{0, p}$ generate the inhomogeneous slider

$$
V_{0, p}=\left\{\omega_{0, p}, v_{0, p}+\omega_{0, p} \times r_{p, x}, \forall x \in \Lambda_{p}\right\}
$$

known as kinematic. The corresponding twist defines the reduction ${ }^{4} V_{0, p}^{t w, p}=$ $\operatorname{col}\left\{v_{0, p}^{p}, \omega_{0, p}^{p}\right\}$ (here we use the fact that the vectors $\omega_{0, p}$ and $v_{0, p}$ can be considered as bounded at the point $O_{p}$ ). This reduction is called vector of quasi-velocities while its component $\omega_{0, p}^{p}$ is known as angular quasi-velocity [4, 14].

Lemma 2. There is the following relation [4]

$$
l^{v_{x}, w r, p}=\Theta_{p, x}^{p} V_{0, p}^{t w, p}, \quad \Theta_{p, x}^{p}=\left[\begin{array}{cc}
I & -r_{p, x}^{\times p} \\
r_{p, x}^{\times p} & -\left(r_{p, x}^{\times p}\right)^{2}
\end{array}\right] .
$$

Proof. The relation is true as

$$
l^{v_{x}, w r, p}=\left[\begin{array}{c}
I \\
r_{p, x}^{\times p}
\end{array}\right] v_{x}^{p}=\left[\begin{array}{c}
I \\
r_{p, x}^{\times p}
\end{array}\right]\left(v_{0, p}^{p}-r_{p, x}^{\times p} \omega_{0, p}^{p}\right)=\left[\begin{array}{cc}
I & -r_{p, x}^{\times p} \\
r_{p, x}^{\times p} & -\left(r_{p, x}^{\times p}\right)^{2}
\end{array}\right]\left(\begin{array}{c}
v_{0, p}^{p} \\
\omega_{0, p}^{p}
\end{array}\right) .
$$

\footnotetext{
${ }^{3}$ A rigid body may comprise continuous and pure points.

${ }^{4}$ Hereinafter one shall mark coordinate representations in any coordinate frame, e.g., $\mathcal{E}_{p}$ with the help of the superscript ${ }^{p}$.
} 
According to the rigid body definition the internal constraints are considered as ideal and thus [13]

$$
\mathcal{R}_{\text {int }}\left(\Lambda_{t}\right)=0 .
$$

From relations (6 [2]) and (2) follows that (see Appendix B [2])

$$
\int \chi_{\Lambda_{t}}\left(\rho_{x} \frac{d}{d t}+\nu_{x}\right) l^{v_{x}, w r, 0} \mu_{3}(d x)=\int \chi_{\Lambda_{t}} l^{f_{x}+\xi_{x}, w r, 0} \mu_{3}(d x)
$$

or

$$
\begin{aligned}
\int \chi_{\Lambda_{p}} L_{0, p}^{w r}\left[\rho_{x}\left(\Theta_{p, x}^{p} \frac{d}{d t}+\frac{d}{d t} \Theta_{p, x}^{p}+\Phi_{0, p}^{w r} \Theta_{p, x}^{p}\right)+\nu_{x} \Theta_{p, x}^{p}\right] V_{0, p}^{t w, p} \mu_{3}(d x) \\
=\int \chi_{\Lambda_{p}} l^{f_{x}+\xi_{x}, w r, 0} \mu_{3}(d x),
\end{aligned}
$$

where the matrices $L_{0, p}^{w r}$ and $\Phi_{0, p}^{w r}$ are defined in Appendix A.

As the twist reduction $V_{0, p}^{t w, p}$ and the matrices $L_{0, p}^{w r}$ and $\Phi_{0, p}^{w r}$ do not depend on points $x \in \Lambda_{p}$ and the matrix $\Theta_{p, x}^{p}$ is time-invariant, the following statement is true.

Theorem 1. The motion of $\alpha_{p}$ (w.r.t. $\mathcal{E}_{0}$ in the frame $\mathcal{E}_{p}$ ) is described by the (NewtonEuler) equation in quasi-velocities [4]

$$
\Theta_{\rho}^{p} \frac{d}{d t} V_{0, p}^{t w, p}+\left(Q_{\nu}^{p}+\Phi_{0, p}^{w r} \Theta_{\rho}^{p}\right) V_{0, p}^{t w, p}=\mathcal{F}^{w r, p},
$$

where

$\Theta_{\rho}^{p}=\int \chi_{\Lambda_{p}} \Theta_{p, x}^{p} \rho_{x} \mu_{3}(d x), Q_{\nu}^{p}=\int \chi_{\Lambda_{p}} \Theta_{p, x}^{p} \nu_{x} \mu_{3}(d x), \mathcal{F}^{w r, p}=\int \chi_{\Lambda_{p}}{ }^{f_{x}+\xi_{x}, w r, p} \mu_{3}(d x)$.

It is easy to see that the matrices of relation (4) depend on the rotation matrix (and translation and angular quasi-velocities, too). That is why equation (4) must be considered along with the Poisson kinematic relation - see below (6).

We may reduce the order of this system by using various parametric representations of rotation matrices [4].

Remark 4. Systems of consecutively connected rigid bodies are considered in [15].

\section{Continuum}

Cauchy continuum. We shall assume that all points of $\Lambda_{t} \subset \tilde{\Lambda}_{t}$ are continuous.

Introduce so-called (homogeneous) measure $\mathcal{F}$ of mass action having the values

$$
\mathcal{F}\left(\Lambda_{t}\right)=\int \chi_{\Lambda_{t}} \rho_{x} l^{g_{x}, w r} \mu_{3}(d x), \quad \Lambda_{t} \subset \tilde{\Lambda}_{t}, t \in \mathbf{T}
$$


on the sets $\Lambda_{t}$ with the density $g_{x}$.

Due to [7] constraints being in a small vicinity of $x \in \Lambda_{t}$ cause the measure $\mathcal{R}_{\text {int }}$ of contact action or stress having the values [3]

$$
\mathcal{R}_{\text {int }}\left(\Lambda_{t}\right)=\int \chi_{\partial \Lambda_{t}} l^{P_{x}, w r} n_{x} \mu_{2}(d x), \quad \Lambda_{t} \subset \tilde{\Lambda}_{t}, t \in \mathbf{T}
$$

on the sets $\Lambda_{t}$ (here $P_{x}$ is stress tensor).

The mechanical system $\alpha=\left\{\Lambda_{t} \subset \tilde{\Lambda}_{t}, \mu_{3}, \nu_{x}, \rho_{x}, \mathcal{F}, \mathcal{R}_{\text {ext }}, \mathcal{R}_{\text {int }}, \Delta \mathcal{P}, \forall t \in \mathbf{T}\right\}$, satisfying to relation (6 [2]) with homogeneous screw measures $\mathcal{P}\left(\Lambda_{t}\right), \mathcal{F}, \mathcal{R}_{\text {ext }}$, $\mathcal{R}_{\text {int }}$ and $\Delta \mathcal{P}$, is called Cauchy continuous medium or continuum.

For the sake of brevity assume that $\Delta \mathcal{P}$ and $\mathcal{R}_{\text {ext }} \equiv 0$. Then due to the lemma 1 from relation (6, [2]) follows (see Appendix B [2])

$$
\left(\rho_{x} \frac{d}{d t}+\nu_{x}\right) v_{x}=\rho_{x} g_{x}+\operatorname{div} P_{x}, \quad P_{x}^{T}=P_{x}
$$

and thus the stress tensor $P_{x}$ has to be symmetric.

Continuum of Hooke class. As we will further use the divergence of $S_{x}$ and $S_{x}$, we do not take into account their skew parts in its calculation. Denote the symmetric tensors $\frac{1}{2}\left(S_{x}+S_{x}^{T}\right)$ or $\frac{1}{2}\left(S_{x}^{\cdot}+S_{x}^{\cdot T}\right)$ as $Z_{x}$.

Definition 5. The Cauchy continuum is called continuum of Hooke class if the tensor $P_{x}$ is a linear isotropic map of $Z_{x}$, i.e., invariant w.r.t. Galilean group of transformations (see also Appendix A).

\section{Conclusion}

We formulated axioms of rational mechanics and examined the question of their implementation on examples of various types of Cosserat-Zhilin systems.

The author would be highly grateful with whoever would bring any element likely to be able to make progress the development, and thus the comprehension, of the paper. Any comments, reviews, critiques, or objections are kindly invited to be sent to the author by e-mail.

\section{APPENDICES}

\section{A The Generalized Galilean GROUP}

Consider two Cartesian frames $\mathcal{E}_{0}$ and $\mathcal{E}_{p}$ in $\mathbf{R}^{3}$ with bases $\mathbf{e}_{0}$ and $\mathbf{e}_{p}$, respectively. We shall assume that the former is immobile while the latter can move w.r.t. the former. Introduce the radius-vectors $r_{x}$ and $r_{p, x}$ of a point $x \in \mathbf{R}^{3}$ w.r.t. the origins $O_{0}$ and $O_{p}$, respectively. Define the vector $d_{0, p}=r_{x}-r_{p, x}$. The vector $v_{x}=r_{x}^{\cdot}$ is 
the velocity of $x$ w.r.t. $O_{0}$ while the vector $v_{0, p}=d_{0, p}$ is translation velocity of $\mathcal{E}_{p}$ w.r.t. $O_{0}$.

There exists a unique vector $\omega_{0, p}$ such that [16]

$$
v_{x}=v_{0, p}+\omega_{0, p} \times r_{p, x}, \quad \forall x \in \mathbf{R}^{3} .
$$

We may represent the relation $r_{x}=d_{0, p}+r_{p, x}$ in the coordinate frame $\mathcal{E}_{0}$ as $r_{x}^{0}=d_{0, p}^{0}+C_{0, p} r_{p, x}^{p}$. With differentiating the above relation we have

$$
v_{x}^{0}=v_{0, p}^{0}+C_{0, p}^{\cdot} r_{p, x}^{p}=v_{0, p}^{0}+C_{0, p}^{\cdot} C_{p, 0} r_{p, x}^{0} .
$$

In the coordinate frame $\mathcal{E}_{p}$ we have $v_{x}^{p}=v_{0, p}^{p}+C_{p, 0} C_{0, p}^{\cdot} p_{p, x}^{p}$. Thus the entries of the cross-product matrix

$$
\omega_{0, p}^{\times p} \stackrel{\text { def }}{=} C_{p, 0} C_{0, p}^{\cdot}
$$

define the coordinates of $\omega_{0, p}$ in $\mathcal{E}_{p}$.

From (5) follows the Poisson kinematic relation

$$
C_{0, p}^{\cdot}=C_{0, p} \omega_{0, p}^{\times p}
$$

Introduce the following matrices:

(7) $C_{0, p}^{\oplus}=\left[\begin{array}{cc}C_{0, p} & O \\ O & C_{0, p}\end{array}\right], \quad D_{0, p}^{0}=\left[\begin{array}{cc}I & O \\ d_{0, p}^{\times 0} & I\end{array}\right], \quad D_{0, p}^{p}=\left[\begin{array}{cc}I & O \\ d_{0, p}^{\times p} & I\end{array}\right]$,

where $I$ is the unit matrix, $O$ is the zero one.

Theorem 2. Let $\mathbf{H}$ be the set of all sliders. A given inhomogeneous slider $l \in \mathbf{H}$

$$
l^{w r, 0}=L_{0, p}^{w r} l^{w r, p},
$$

where $l^{w r, 0}$ and $l^{w r, p}$ are wrench reductions of the slider $l$ computed in the bases $\mathbf{e}_{0}$ and $\mathbf{e}_{p}$, respectively, the matrix $L_{0, p}^{w r}$ is defined by the following relation [4]:

$$
L_{0, p}^{w r}=C_{0, p}^{\oplus} D_{0, p}^{p}=D_{0, p}^{0} C_{0, p}^{\oplus}
$$

and belongs to the multiplicative group $\mathcal{L}^{\text {wr }}$ such that

(9) $\quad L_{0, p}^{w r .}=L_{0, p}^{w r} \Phi_{0, p}^{w r}=\Psi_{0, p}^{w r} L_{0, p}^{w r}, \Phi_{0, p}^{w r}=\left[\begin{array}{cc}\omega_{0, p}^{\times p} & O \\ v_{0, p}^{\times p} & \omega_{0, p}^{\times p}\end{array}\right], \Psi_{0, p}^{w r}=\left[\begin{array}{cc}\omega_{0, p}^{\times 0} & O \\ v_{0, p}^{\times 0} & \omega_{0, p}^{\times 0}\end{array}\right]$. 
Implementation of the Axioms of Cosserat-Zhilin Systems on Examples of... 11

Proof. Relation (8) follows directly from the slider definition.

Consider the case where $L_{0, p}^{w r}=C_{0, p}^{\oplus} D_{0, p}^{p}$. Then from (7) follows:

$$
\begin{aligned}
L_{0, p}^{w r} & =C_{0, p}^{\oplus \cdot} D_{0, p}^{p}+C_{0, p}^{\oplus} D_{0, p}^{p .} \\
& =\left(C_{0, p}^{\oplus \cdot} D_{0, p}^{p} D_{p, 0}^{p} C_{p, 0}^{\oplus}+C_{0, p}^{\oplus} D_{0, p}^{p \cdot} D_{p, 0}^{p} C_{p, 0}^{\oplus}\right) C_{0, p}^{\oplus} D_{0, p}^{p} \\
& =\left(C_{0, p}^{\oplus} C_{0, p}^{\oplus}+D_{0, p}^{0 .}\right) C_{0, p}^{\oplus} D_{0, p}^{p}=\Psi_{0, p}^{w r} L_{0, p}^{w r} .
\end{aligned}
$$

In the case where $L_{0, p}^{w r}=D_{0, p}^{0} C_{0, p}^{\oplus}$, from (7) follows:

$$
\begin{aligned}
L_{0, p}^{w r .} & =D_{0, p}^{0 .} C_{0, p}^{\oplus}+D_{0, p}^{0} C_{0, p}^{\oplus .} \\
& =D_{0, p}^{0} C_{0, p}^{\oplus}\left(C_{p, 0}^{\oplus} C_{0, p}^{\oplus \cdot}+C_{p, 0}^{\oplus} D_{p, 0}^{0} D_{0, p}^{0 .} C_{0, p}^{\oplus}\right) \\
& =L_{0, p}^{w r} \Phi_{0, p}^{w r} .
\end{aligned}
$$

Thus we have relation (9).

Let $C_{p, k}$ be the rotation matrix of a Cartesian frame $\mathcal{E}_{k}$ w.r.t. $\mathcal{E}_{p}$. Then

$$
\begin{aligned}
L_{0, p}^{w r} L_{p, k}^{w r} & =C_{0, p}^{\oplus} C_{p, k}^{\oplus} C_{k, p}^{\oplus} D_{0, p}^{p} C_{p, k}^{\oplus} D_{p, k}^{k} \\
& =C_{0, k}^{\oplus} D_{0, p}^{k} D_{p, k}^{k}=C_{0, k}^{\oplus} D_{0, k}^{k}=L_{0, k}^{w r}
\end{aligned}
$$

and

$$
\begin{aligned}
L_{0, p}^{w r,-1} & =\left(C_{0, p}^{\oplus} D_{0, p}^{p}\right)^{-1}=\left(D_{0, p}^{p}\right)^{-1} C_{0, p}^{\oplus, T} \\
& =D_{p, 0}^{p} C_{p, 0}^{\oplus}=C_{p, 0}^{\oplus} D_{p, 0}^{0}=L_{p, 0}^{w r},
\end{aligned}
$$

i.e., matrices of the kind $L_{0, p}^{w r}=C_{0, p}^{\oplus} D_{0, p}^{p}$ form a multiplicative group.

The similar statement $l^{t w, p}=L_{0, p}^{t w} l^{t w, 0}$ is true for twists where we have the matrix

$$
L_{0, p}^{t w}=\left[\begin{array}{cc}
O & I \\
I & O
\end{array}\right] L_{0, p}^{w r}\left[\begin{array}{cc}
O & I \\
I & O
\end{array}\right]
$$

belongs to the multiplicative group $\mathcal{L}^{t w}$ such that $L_{0, p}^{t w}=\Psi_{0, p}^{t w} L_{0, p}^{t w}, \Psi_{0, p}^{t w}=-\Psi_{0, p}^{w r, T}$ and $L_{0, p}^{t w}=\Phi_{0, p}^{t w} L_{0, p}^{t w}, \Phi_{0, p}^{t w}=-\Phi_{0, p}^{w r, T}$

The groups $\mathcal{L}^{w r}$ and $\mathcal{L}^{t w}$ generate the group $\mathcal{L}(\mathbf{H}, 6)$ acting in the slider set $\mathbf{H}$ when there is a change of coordinate frames, e.g., from $\mathcal{E}_{0}$ to $\mathcal{E}_{p}$.

Definition 6. The group $\mathcal{G} \mathcal{L}(\mathbf{H}, 6) \subset \mathcal{L}(\mathbf{H}, 6)$ is called generalized Galilean group if the translation vector $d_{0, p}$ is replaced with $d_{0, p}+v_{0, p} t$, the initial translation $d_{0, p}$ and the translation velocity $v_{0, p}$ of $\mathcal{E}_{p}$ w.r.t. $\mathcal{E}_{0}$ are constant while the angular velocity $\omega_{0, p}$ is constant, too [4]. 


\section{B MultiPliCATIVE GROUPS OF LINEAR ISOTROPIC MAPS}

3-dimensional case. Given any $3 \times 3$-matrix $Z$, let us define the linear independent matrices

$$
E_{1}=(\operatorname{trace} Z) I, E_{2}=Z, E_{2}=Z^{T} .
$$

Aggregates $A E_{i} B(i=\overline{1,3})$ are isotropic functions of $Z$ entries if the matrices $A$ and $B$ are proportional to $I$ with scalar coefficients being invariant w.r.t. Galilean group of transformations. Hereinafter all scalar coefficients used below will be considered as invariant w.r.t. this group.

Let us construct the sets of all isotropic functions [17]

$P=p_{0} I+p_{1}(\operatorname{trace} Z) I+p_{2} Z+p_{3} Z^{T}, \quad Q=q_{0} I+q_{1}(\operatorname{trace} P) I+q_{2} P+q_{3} P^{T}$

where $p_{i}$ and $q_{i}$ are scalar coefficients.

Then

$$
\begin{aligned}
Q= & q_{0} I+q_{1}\left[3 p_{0}+\left(3 p_{1}+p_{2}+p_{3}\right) \operatorname{trace} Z\right] I \\
& +q_{2}\left[p_{0} I+p_{1}(\operatorname{trace} Z) I+p_{2} Z+p_{3} Z^{T}\right] \\
& +q_{3}\left[p_{0} I+p_{1}(\operatorname{trace} Z) I+p_{2} Z^{T}+p_{3} Z\right] \\
= & \left(q_{0}+3 p_{0} q_{1}+p_{0} q_{2}+p_{0} q_{3}\right) I \\
& +\left[\left(3 p_{1}+p_{2}+p_{3}\right) q_{1}+p_{1} q_{2}+\left(p_{2} q_{2}+p_{3} q_{3}\right) Z\right. \\
& \left.+\left(p_{3} q_{2}+p_{2} q_{3}\right) Z^{T}+p_{1} q_{3}\right](\operatorname{trace} Z) I \\
& +r_{0} I+r_{1}(\operatorname{trace} Z) I+r_{2} Z+r_{3} Z^{T}
\end{aligned}
$$

where

$$
\left(\begin{array}{l}
r_{0} \\
r_{1} \\
r_{2} \\
r_{3}
\end{array}\right)=R\left(\begin{array}{l}
q_{0} \\
q_{1} \\
q_{2} \\
q_{3}
\end{array}\right), \quad R=\left[\begin{array}{cccc}
1 & 3 p_{0} & p_{0} & p_{0} \\
0 & 3 p_{1}+p_{2}+p_{3} & p_{1} & p_{1} \\
0 & 0 & p_{2} & p_{3} \\
0 & 0 & p_{3} & p_{2}
\end{array}\right]
$$

The set of non-singular matrices of the kind $R$ forms a multiplicative group. That is why if $\operatorname{det} R=\left(3 p_{1}+p_{2}+p_{3}\right)\left(p_{2}^{2}-p_{3}^{2}\right) \neq 0$ then in the case where $Q=Z$ we 
have

$$
\left(\begin{array}{l}
q_{0} \\
q_{1} \\
q_{2} \\
q_{3}
\end{array}\right)=R^{-1}\left(\begin{array}{l}
0 \\
0 \\
1 \\
0
\end{array}\right)=\left(\begin{array}{c}
\frac{-p_{0}}{3 p_{1}+p_{2}+p_{3}} \\
\frac{-p_{1}}{\left(p_{2}+p_{3}\right)\left(3 p_{1}+p_{2}+p_{3}\right)} \\
\frac{p_{2}}{p_{2}^{2}-p_{3}^{2}} \\
\frac{-p_{3}}{p_{2}^{2}-p_{3}^{2}}
\end{array}\right)
$$

and the inverse function $Z=q_{0} I+q_{1}(\operatorname{trace} P) I+q_{2} P+q_{3} P^{T}$.

Remark 5. Instead (11) we might choose an other set of linear independent matrices, e.g., $E_{1}=(\operatorname{trace} Z) I, E_{2}=\frac{1}{2}\left(Z+Z^{T}\right), E_{2}=\frac{1}{2}\left(Z-Z^{T}\right)$.

In the case where the matrix $Z$ is symmetric let us define the linear independent matrices $E_{1}=(\operatorname{trace} Z) I, E_{2}=Z$ and the sets of linear isotropic functions $P=p_{0} I+p_{1}(\operatorname{trace} Z) I+r_{2} Z, Q=q_{0} I+q_{1}(\operatorname{trace} P) I+q_{2} P$. As trace $P=$ $3 p_{0}+\left(3 p_{1}+p_{2}\right)$ trace $Z$ we have $Q=\left(q_{0}+3 p_{0} q_{1}+p_{0} q_{2}\right) I+\left[\left(3 p_{1}+p_{2}\right) q_{1}+\right.$ $\left.p_{1} q_{2}\right](\operatorname{trace} Z) I+q_{2}\left[p_{0} I+p_{1}(\operatorname{trace} Z) I+r_{2} Z\right]$ or $Q=r_{0} I+r_{1}(\operatorname{trace} Z) I+r_{2} Z$ where

$$
\left(\begin{array}{l}
r_{0} \\
r_{1} \\
r_{2}
\end{array}\right)=R\left(\begin{array}{l}
q_{0} \\
q_{1} \\
q_{2}
\end{array}\right), \quad R=\left[\begin{array}{ccc}
1 & 3 p_{0} & p_{0} \\
0 & 3 p_{1}+p_{2} & p_{1} \\
0 & 0 & p_{2}
\end{array}\right] .
$$

Thus under the condition that $\operatorname{det} R=\left(3 p_{1}+p_{2}\right) p_{2} \neq 0$ for the case where $Q=Z$ we have

$$
\left(\begin{array}{l}
q_{0} \\
q_{1} \\
q_{2}
\end{array}\right)=R^{-1}\left(\begin{array}{l}
0 \\
0 \\
1
\end{array}\right)=\left(\begin{array}{c}
\frac{-p_{0}}{3 p_{1}+p_{2}} \\
\frac{-p_{1}}{p_{2}\left(3 p_{1}+p_{2}\right)} \\
\frac{1}{p_{2}}
\end{array}\right)
$$

and the inverse function $Z=q_{0} I+q_{1}(\operatorname{trace} P) I+q_{2} P$.

2-dimensional case. Since the formulation of the axioms of mechanics can be restated in the two-dimensional space and Lemma 1 can be reformulated with using Green's theorem, let us consider the multiplicative group of isotropic maps for 2dimensional media.

Given $2 \times 2$-matrix $Z=\left[\begin{array}{ll}a & b \\ c & d\end{array}\right]$ let us define the matrices $E_{1}=(\operatorname{trace} Z) I$, $E_{2}=(\operatorname{trace} \widetilde{I} Z) I, E_{3}=Z, E_{4}=\widetilde{I} Z, E_{5}=Z^{T}, E_{6}=Z^{T} \widetilde{I}, E_{7}=\widetilde{I} Z^{T}$, 
$E_{8}=Z \widetilde{I}, E_{9}=\widetilde{I} Z \widetilde{I}$ and $E_{10}=\widetilde{I} Z^{T} \widetilde{I}$, where $I$ is the identity $2 \times 2$ matrix, $\widetilde{I}=\left[\begin{array}{cc}0 & -1 \\ 1 & 0\end{array}\right]$.

It is plain that the first 6 matrices $E_{1}, E_{2}, E_{3}, E_{4}, E_{5}$ and $E_{6}$ are linear independent and $E_{7}=E_{4}-E_{2}, E_{8}=E_{2}+E_{6}, E_{9}=-E_{1}+E_{5}, E_{10}=-E_{1}+E_{3}$.

Aggregates $A E_{i} B$ are isotropic maps of $Z$ entries if $A$ and $B$ are of the kind $\alpha I+\beta \widetilde{I}$ where $\alpha$ and $\beta$ are scalar coefficients.

Introduce the sets of isotropic functions

$$
\begin{aligned}
& P=p_{0} I+\widetilde{p}_{0} \widetilde{I}+p_{1}(\operatorname{trace} Z) I+p_{2}(\operatorname{trace} \widetilde{I} Z) I+p_{3} Z+p_{4} Z^{T}+p_{5} \widetilde{I} Z+p_{6} Z^{T} \widetilde{I}, \\
& Q=q_{0} I+\widetilde{q}_{0} \widetilde{I}+q_{1}(\operatorname{trace} P) I+q_{2}(\operatorname{trace} \widetilde{I} P) I+q_{3} P+q_{4} P^{T}+q_{5} \widetilde{I} P+q_{6} P^{T} \widetilde{I} .
\end{aligned}
$$

Then

$$
\begin{aligned}
\operatorname{trace} P & =2 p_{0}+\left(2 p_{1}+p_{3}+p_{4}\right) \operatorname{trace} Z+\left(2 p_{2}+p_{5}-p_{6}\right) \operatorname{trace} \widetilde{I} Z, \\
\widetilde{I} P & =p_{0} \widetilde{I}-\widetilde{p}_{0} I+p_{1}(\operatorname{trace} Z) \widetilde{I}+p_{2}(\operatorname{trace} \widetilde{I} Z) \widetilde{I}+p_{3} \widetilde{I} Z+p_{4} \widetilde{I} Z \\
& -p_{5} Z+p_{6} \widetilde{I} Z^{T} \widetilde{I}, \\
\operatorname{trace} \widetilde{I} P & =-2 \widetilde{p}_{0}-\left(p_{5}+p_{6}\right) \operatorname{trace} Z+\left(p_{3}-p_{4}\right) \operatorname{trace} \widetilde{I} Z, \\
P^{T} \widetilde{I} & =p_{0} \widetilde{I}+\widetilde{p}_{0} I+p_{1}(\operatorname{trace} Z) \widetilde{I}+p_{2}(\operatorname{trace} \widetilde{I} Z) \widetilde{I}+p_{3} Z^{T} \widetilde{I}+p_{4} Z \widetilde{I} \\
& +p_{5} Z^{T}-p_{6} \widetilde{I} Z \widetilde{I} .
\end{aligned}
$$

As $\widetilde{I} Z^{T}=\widetilde{I} Z-(\operatorname{trace} \widetilde{I} Z) I, Z \widetilde{I}=(\operatorname{trace} \widetilde{I} Z) I+Z^{T} \widetilde{I}, \widetilde{I} Z \widetilde{I}=Z^{T}-(\operatorname{trace} Z) I$, $\widetilde{I} Z^{T} \widetilde{I}=Z-(\operatorname{trace} Z) I, \operatorname{trace} Z=a+d, \operatorname{trace} \widetilde{I} Z=b-c$, trace $\widetilde{I} Z^{T}=-(b-c)$, $(\operatorname{trace} Z) \widetilde{I}=\widetilde{I} Z+Z^{T} \widetilde{I}, \operatorname{trace} \widetilde{I} Z=-\operatorname{trace} Z^{T} \widetilde{I}$, $\operatorname{trace} Z \widetilde{I}=\operatorname{trace} \widetilde{I} Z=b-c$, $(\operatorname{trace} Z \widetilde{I}) \widetilde{I}=Z^{T}-Z,(\operatorname{trace} \widetilde{I} Z) \widetilde{I}=Z^{T}-Z=(\operatorname{trace} Z \widetilde{I}) \widetilde{I}, \operatorname{trace} \widetilde{I} Z^{T} \widetilde{I}=$ $\operatorname{trace} \widetilde{I} Z \widetilde{I}=-\operatorname{trace} Z$ we have

$$
\begin{aligned}
Q= & {\left[2 p_{0} q_{1}+\left(2 p_{1}+p_{3}+p_{4}\right) q_{1} \operatorname{trace} Z+\left(2 p_{2}+p_{5}-p_{6}\right) q_{1}(\operatorname{trace} \widetilde{I} Z)\right] I } \\
& +q_{0} I+\widetilde{q_{0}} \widetilde{I}+\left[-2 \widetilde{p}_{0} q_{2}-\left(p_{5}+p_{6}\right) q_{2}(\operatorname{trace} Z)+\left(p_{3}-p_{4}\right) q_{2}(\operatorname{trace} \widetilde{I} Z)\right] I \\
& +p_{0} q_{3} I+\widetilde{p}_{0} q_{3} \widetilde{I}+p_{1} q_{3}(\operatorname{trace} Z) I+p_{2} q_{3}(\operatorname{trace} \widetilde{I} Z) I+p_{3} q_{3} Z+p_{4} q_{3} Z^{T} \\
& +p_{5} q_{3} \widetilde{I} Z+p_{6} q_{3} Z^{T} \widetilde{I}+p_{0} q_{4} I-\widetilde{p}_{0} q_{4} \widetilde{I}+p_{1} q_{4}(\operatorname{trace} Z) I+p_{2} q_{4}(\operatorname{trace} \widetilde{I} Z) I \\
& +p_{4} q_{4} Z+p_{3} q_{4} Z^{T}-p_{6} q_{4} \widetilde{I} Z-p_{5} q_{4} Z^{T} \widetilde{I}+p_{0} q_{5} \widetilde{I}-\widetilde{p}_{0} q_{5} I-p_{6} q_{5}(\operatorname{trace} Z) I \\
& -p_{4} q_{5}(\operatorname{trace} \widetilde{I} Z) I+\left(p_{6}-p_{5}-p_{2}\right) q_{5} Z+p_{2} q_{5} Z^{T}+\left(p_{1}+p_{3}+p_{4}\right) q_{5} \widetilde{I} Z \\
& +p_{1} q_{5} Z^{T} \widetilde{I}+p_{0} q_{6} \widetilde{I}+\widetilde{p}_{0} q_{6} I+p_{6} q_{6}(\operatorname{trace} Z) I+p_{4} q_{6}(\operatorname{trace} \widetilde{I} Z) I-p_{2} q_{6} Z \\
& +\left(p_{2}+p_{5}-p_{6}\right) q_{6} Z^{T}+p_{1} q_{6} \widetilde{I} Z+\left(p_{1}+p_{3}+p_{4}\right) q_{6} Z^{T} \widetilde{I}
\end{aligned}
$$


Implementation of the Axioms of Cosserat-Zhilin Systems on Examples of... 15

or

$$
\begin{aligned}
Q= & \left(q_{0}+2 p_{0} q_{1}-2 \widetilde{p}_{0} q_{2}+p_{0} q_{3}+p_{0} q_{4}-\widetilde{p}_{0} q_{5}+\widetilde{p}_{0} q_{6}\right) I \\
& +\left(\widetilde{p}_{0}+\widetilde{p}_{0} q_{3}-\widetilde{p}_{0} q_{4}+p_{0} q_{5}+p_{0} q_{6}\right) \widetilde{I} \\
& +\left[\left(2 p_{1}+p_{3}+p_{4}\right) q_{1}-\left(p_{5}+p_{6}\right) q_{2}+p_{1} q_{3}+p_{1} q_{4}-p_{6} q_{5}+p_{6} q_{6}\right] \operatorname{trace} Z \\
& +\left[\left(2 p_{2}+p_{5}-p_{6}\right) q_{1}+\left(p_{3}-p_{4}\right) q_{2}+p_{2} q_{3}+p_{2} q_{4}-p_{4} q_{5}+p_{4} q_{6}\right] \operatorname{trace} \widetilde{I} Z \\
& +\left[p_{3} q_{3}+p_{4} q_{4}+\left(p_{6}-p_{5}-p_{2}\right) q_{5}-p_{2} q_{6}\right] Z \\
& +\left[p_{4} q_{3}+p_{3} q_{4}+p_{2} q_{5}+\left(p_{2}+p_{5}-p_{6}\right) q_{6}\right] Z^{T} \\
& +\left[p_{5} q_{3}-p_{6} q_{4}+\left(p_{1}+p_{3}+p_{4}\right) q_{5}+p_{1} q_{6}\right] \widetilde{I} Z \\
& +\left[p_{6} q_{3}-p_{5} q_{4}+p_{1} q_{5}+\left(p_{1}+p_{3}+p_{4}\right) q_{6}\right] Z^{T} \widetilde{I} \\
= & r_{0} I+\widetilde{r}_{0} \widetilde{I}+r_{1}(\operatorname{trace} Z) I+r_{2}(\operatorname{trace} \widetilde{I} Z) I+r_{3} Z+r_{4} Z^{T}+r_{5} \widetilde{I} Z+r_{6} Z^{T} \widetilde{I} .
\end{aligned}
$$

Hence $\operatorname{col}\left\{r_{0}, \widetilde{r}_{0}, r_{1}, r_{2}, r_{3}, r_{4}, r_{4}, r_{5}, r_{6}\right\}=R \operatorname{col}\left\{q_{0}, \widetilde{q}_{0}, q_{1}, q_{2}, q_{3}, q_{4}, q_{4}, q_{5}, q_{6}\right\}$ with

$$
R=\left[\begin{array}{cccccccc}
1 & 0 & 2 p_{0} & -2 \widetilde{p}_{0} & p_{0} & p_{0} & -\widetilde{p}_{0} & \widetilde{r}_{0} \\
0 & 1 & 0 & 0 & \widetilde{p}_{0} & -\widetilde{p}_{0} & p_{0} & p_{0} \\
0 & 0 & 2 p_{1}+p_{3}+p_{4} & -\left(p_{5}+p_{6}\right) & p_{1} & p_{1} & -p_{6} & p_{6} \\
0 & 0 & 2 p_{2}+p_{5}-p_{6} & p_{3}-p_{4} & p_{2} & p_{2} & -p_{4} & p_{4} \\
0 & 0 & 0 & 0 & p_{3} & p_{4} & p_{6}-p_{5}-p_{2} & -p_{2} \\
0 & 0 & 0 & 0 & p_{4} & p_{3} & p_{2} & p_{2}+p_{5}-p_{6} \\
0 & 0 & 0 & 0 & p_{5} & -p_{6} & p_{1}+p_{3}+p_{4} & p_{1} \\
0 & 0 & 0 & 0 & p_{6} & -p_{5} & p_{1} & p_{1}+p_{3}+p_{4}
\end{array}\right] .
$$

It is plain that $\operatorname{det} R=-4\left[\left(2 p_{1}+p_{3}+p_{4}\right)\left(p_{3}-p_{4}\right)+\left(2 p_{2}+p_{5}-p_{6}\right)\left(p_{5}+p_{6}\right)\right]^{2}\left[\left(p_{3}+\right.\right.$ $\left.\left.p_{4}\right)^{2}+\left(p_{5}-p_{6}\right)^{2}\right]$. Under the condition that $\operatorname{det} R \neq 0$ in the case where $Q=Z$ we have

$$
\begin{aligned}
\left(\begin{array}{l}
q_{0} \\
\widetilde{q}_{0} \\
q_{1} \\
q_{2}
\end{array}\right)= & -\left[\begin{array}{llcc}
1 & 0 & 2 p_{0} & -2 \widetilde{p}_{0} \\
0 & 1 & 0 & 0 \\
0 & 0 & 2 p_{1}+p_{3}+p_{4} & -\left(p_{5}+p_{6}\right) \\
0 & 0 & 2 p_{2}+p_{5}-p_{6} & p_{3}-p_{4}
\end{array}\right]^{-1}\left[\begin{array}{cccc}
p_{0} & p_{0} & -\widetilde{p}_{0} & \widetilde{p}_{0} \\
\widetilde{p}_{0} & -\widetilde{p}_{0} & p_{0} & p_{0} \\
p_{1} & p_{1} & -p_{6} & p_{6} \\
p_{2} & p_{2} & -p_{4} & p_{4}
\end{array}\right]\left(\begin{array}{l}
q_{3} \\
q_{4} \\
q_{5} \\
q_{6}
\end{array}\right) \\
\left(\begin{array}{l}
q_{3} \\
q_{4} \\
q_{5} \\
q_{6}
\end{array}\right)= & {\left[\begin{array}{cccc}
p_{3} & p_{4} & p_{6}-p_{5}-p_{2} & -p_{2} \\
p_{4} & p_{3} & p_{2} & p_{2}+p_{5}-p_{6} \\
p_{5} & -p_{6} & p_{1}+p_{3}+p_{4} & p_{1} \\
p_{6} & -p_{5} & p_{1} & p_{1}+p_{3}+p_{4}
\end{array}\right]^{-1}\left(\begin{array}{l}
1 \\
0 \\
0 \\
0
\end{array}\right) }
\end{aligned}
$$


and the inverse function

$Z=q_{0} I+\widetilde{q}_{0} \widetilde{I}+q_{1}(\operatorname{trace} P) I+q_{2}(\operatorname{trace} \widetilde{I} P) I+q_{3} P+q_{4} P^{T}+q_{5} \widetilde{I} P+q_{6} P^{T} \widetilde{I}$.

If the matrix $Z$ is symmetric we have the linearly independent matrices $E_{1}=\operatorname{trace} Z$, $E_{2}=Z$ and $E_{3}=\widetilde{I} Z-Z \widetilde{I}$. Define the sets of linear isotropic functions

$$
\begin{aligned}
& P=p_{0} I+p_{1}(\operatorname{trace} Z) I+p_{2} Z+p_{3}(\widetilde{I} Z-Z \widetilde{I}), \\
& Q=q_{0} I+q_{1}(\operatorname{trace} P) I+q_{2} P+q_{3}(\widetilde{I} P-P \widetilde{I}) .
\end{aligned}
$$

With the help of the following relations

$$
\begin{aligned}
\widetilde{I} P & =p_{0} \widetilde{I}+p_{1}(\operatorname{trace} Z) \widetilde{I}+p_{2} \widetilde{I} Z-p_{3}(Z+\widetilde{I} Z \widetilde{I}), \\
P \widetilde{I} & =p_{0} \widetilde{I}+p_{1}(\operatorname{trace} Z) \widetilde{I}+p_{2} Z \widetilde{I}+p_{3}(\widetilde{I} Z \widetilde{I}+Z), \\
\widetilde{I} P-P \widetilde{I} & =p_{2}(\widetilde{I} Z-Z \widetilde{I})-2 p_{3}(Z+\widetilde{I} Z \widetilde{I}) \\
& =p_{2}(\widetilde{I} Z-Z \widetilde{I})+2 p_{3}[(\operatorname{trace} Z) I-2 Z] .
\end{aligned}
$$

we have

$$
\begin{aligned}
Q= & q_{0} I+q_{1}\left[2 p_{0}+\left(2 p_{1}+p_{2}\right)(\operatorname{trace} Z)\right] I+ \\
& q_{2}\left[p_{0} I+p_{1}(\operatorname{trace} Z) I+p_{2} Z+p_{3}(\widetilde{I} Z-Z \widetilde{I})\right]+ \\
& q_{3}\left\{p_{2}(\widetilde{I} Z-Z \widetilde{I})+2 p_{3}[(\operatorname{trace} Z) I-2 Z]\right\} \\
= & q_{0} I+2 p_{0} q_{1} I+p_{0} q_{2} I+\left[\left(2 p_{1}+p_{2}\right) q_{1}+p_{1} q_{2}+2 p_{3} q_{3}\right](\operatorname{trace} Z) I+ \\
& \left(p_{2} q_{2}-4 p_{3} q_{3}\right) Z+\left(p_{3} q_{2}+p_{2} q_{3}\right)(\widetilde{I} Z-Z \widetilde{I}) \\
= & r_{0} I+r_{1}(\operatorname{trace} Z) I+r_{2} Z+r_{3}(\widetilde{I} Z-Z \widetilde{I})
\end{aligned}
$$

and $\operatorname{col}\left\{r_{0}, r_{1}, r_{2}, r_{3}\right\}=R \operatorname{col}\left\{q_{0}, q_{1}, q_{2}, q_{3}\right\}$ where

$$
R=\left[\begin{array}{cccc}
1 & 2 p_{0} & p_{0} & 0 \\
0 & 2 p_{1}+p_{2} & p_{1} & 2 p_{3} \\
0 & 0 & p_{2} & -4 p_{3} \\
0 & 0 & p_{3} & p_{2}
\end{array}\right] .
$$

Under the condition that det $R=\left(2 p_{1}+p_{2}\right)\left(p_{2}^{2}+4 p_{3}^{2}\right) \neq 0$, in the case where $Q=Z$ there are $\operatorname{col}\left\{q_{0}, q_{1}, q_{2}, q_{3}\right\}=R^{-1} \operatorname{col}\{0,0,1,0\}$ and the inverse function

$$
Z=q_{0} I+q_{1}(\operatorname{trace} P) I+q_{2} P+q_{3}(\widetilde{I} P-P \widetilde{I}) .
$$

Remark 6. Using these expressions one can enter linear constitutive relations for continuous momentless and moment 2-and 3-dimensional continua which, in the case of their invertibility, form multiplicative groups. It is an open question about the legality of isotropic maps being unwell-defined (see [4]). 


\section{Multiphase continuum as a Cosserat-Zhilin system}

Following to [3] let us consider a multiphase continuum, consisting of $n$ components, which may occur between $m$ chemical reactions (points forming medium assumed to be continuous). During the reaction the proportion of one component decreases, while the other increases. Assume that each particulate consists of $n$ micro-particles (components), so that at each point in space at any given time there are at once all $n$ components, each with a density $\rho_{\alpha}(x, t)(\alpha=1, \ldots, n)$. Then the total density is defined as $\rho_{x}=\sum_{\alpha=1}^{n} \rho_{\alpha}$.

We assume that the center of mass of each micro-particle does not coincide with the center of mass of the particulates. This is the reason to introduce the inertia tensor of particulate components which are recorded, for example in the form of

$$
J_{i j}=\sum_{\alpha=1}^{n} \rho_{\alpha}\left[\left(\sum_{k=1}^{3} z_{k}^{\alpha} z_{k}^{\alpha}\right) \delta_{i j}-z_{i}^{\alpha} z_{j}^{\alpha}\right],
$$

where $z_{k}^{\alpha}$-coordinates micro-particles $\alpha$ with respect to particulates, which are assumed to be constant.

Let $l_{\alpha}=\left(\sum_{k=1}^{3}\left(z_{k}^{\alpha}\right)^{2}\right)^{1 / 2}$ be the length of the radius vector micro-particles $\alpha$ with respect to particulates. Then the first invariant of tensor (12) has the form

$$
J_{x}=\sum_{i=1}^{3} J_{i i}=2 \sum_{\alpha=1}^{n} \rho_{\alpha} l_{\alpha}^{2} .
$$

Denoting by $v_{\alpha}(x, t)$ velocity of each component, determine the rate of velocity of the particulates as the center of mass of micro-particles

$$
v_{x}=\frac{1}{\rho_{x}} \sum_{\alpha=1}^{n} \rho_{\alpha} v_{\alpha} .
$$

Introduce the continuity equation for each component of the multiphase medium

$$
\frac{\partial \rho_{\alpha}}{\partial t}+\operatorname{div}\left(\rho_{\alpha} v_{\alpha}\right)=\gamma_{\alpha}
$$

where $\gamma_{\alpha}=\sum_{I=1}^{m} \nu_{\alpha I} J_{I}$ is called formation of a compound $\alpha$, the value of $\nu_{\alpha I}$ is proportional to the stoichiometric ratio, with which the component $\alpha$ is included in the $I$-th chemical reaction; $J_{I}$ - velocity of chemical reactions.

Summing $n$ equations (13), we arrive at the continuity equation for the density of particulates

$$
\frac{\partial \rho_{x}}{\partial t}+\operatorname{div}\left(\rho_{x} v_{x}\right)=\sum_{\alpha=1}^{n} \gamma_{\alpha}=\sum_{\alpha=1}^{n} \sum_{I=1}^{m} \nu_{\alpha I} J_{I} .
$$


Using (13), we can write the continuity equation for

$$
\frac{d J_{x}}{d t}+J_{x} \operatorname{div} v_{x}=2 \sum_{\alpha=1}^{n} \gamma_{\alpha} l_{\alpha}^{2} \text { or } \frac{\partial J_{x}}{\partial t}+\operatorname{div}\left(J_{x} v_{x}\right)=2 \sum_{\alpha=1}^{n} \gamma_{\alpha} l_{\alpha}^{2} .
$$

Then using Lemma 1 and continuity equations introduced above, from (6, [2]) we have 6-dimensional differential equation of multiphase continuous medium given in [3].

Just the same equation is obtained if we introduce

$$
p_{x}=\rho_{x} v_{x}, \quad q_{x}=J_{x} \mu_{x},
$$

i.e., the multiphase system proves to be a Cosserat-Zhilin system of the rather general type.

\section{REFERENCES}

[1] A. Einstein (1927) Newton's Mechanik und ihr Einflu auf die Gestaltung der theoretischen Physik. Die Naturwissenschaften 15273276.

[2] Al. Cheremensky (2018) Mechanical Systems of Cosserat-Zhilin. J. Theor. Appl. Mech. (Sofia) 48(4) 3-18; DOI 10.2478/jtam-2018-0019.

[3] B.E. Pobedria, D.V. Georgievsky (2006) "Bases of Continuum Mechanics. Lecture Course". FML, Moscow (in Russian).

[4] V.A. Konoplev (1999) "Algebraic Methods in Galilean Mechanics". Nauka, St. Petersburg; http://mechanics-konoplev.com.

[5] C. Truesdell (1991) "A First Course in Rational Continuum Mechanics". Pure and Applied Mathematics 71 Academic Press, Boston-Toronto.

[6] P.A. ZhiLin (2012) "Rational Continuum Mechanics". Politechnic University Publishing House, St. Petersburg (available at P.A. Zhilin).

[7] N.A. Kilchevsky, G.A. Kilchinsky, N.E. Tkachenko (1979) "Analytical Mechanics of Continua". Naukova Dumka, Kiev (in Russian).

[8] E. Cosserat, F. Cosserat (1909) "Theory of Deformable Bodies". Hermann, Paris.

[9] S.V. VAlLANDER (1978) "Lectures on Hydroaeromechanics". LGUpress, Leningrad (in Russian).

[10] A.I. LuRIE (2005) "Theory of Elasticity". Springer-Verlag, Berlin.

[11] W. NowACKI (1970) "Teoria Sprezystosci”. Panstwowe Wydawnictwo Naukowe, Warszawa (in Polish).

[12] V.F. ZhuraVlev (2001) "Bases of Theoretical Mechanics". IFML, Moscow (in Russian).

[13] V.G. VilKe (2003) “Theoretical Mechanics”. Lan', St. Petersburg (in Russian).

[14] A.I. LuRIE (2002) “Analytical Mechanics". Springer Verlag, Berlin. 
Implementation of the Axioms of Cosserat-Zhilin Systems on Examples of... 19

[15] V.A. Konoplev, A. Cheremensky, On kinematics of multibody systems, C. R. Acad. Bulg. Sci., 2010, 63, n. 9, 1251-1256.

[16] G.N. Yakovenko (2005) "Brief Course on Theoretical Mechanics". Binom, Moscow (in Russian).

[17] B.A. Dubrovin, A.T. Fomenko, S.P. Novikov (1992) Modern Geometry - Methods and Applications. Part I. The Geometry of Surfaces, Transformation Groups, and Fields, trans. by R.G. Burns, 2nd ed. Graduate Texts in Mathematics 93, New York, Springer Verlag. 